\title{
Research Paper \\ Demographic Characteristics and Common Complaints of People Seeking Telephone Consulting Services for Anxiety and Stress Caused by COVID-19
}

\section{Faraj Hoseinian Sarajloo ${ }^{1}$, Roza Rashedi $^{1}$, Haleh Fateh ${ }^{1}$, Hamidreza Shahbazpour ${ }^{1}$, ${ }^{*}$ Bahareh Kashani Movahhed $^{1}$ (C)}

Received: 25 Apr 2020 Accepted: 26 May 2020 Available Online: 01 Jun 2020

Keywords:

Anxiety, Stress, COVID-19, Telephone counseling

\section{ABSTRACT}

Background The Coronavirus Disease 2019 (COVID-19) pandemic and imposed quarantine have had different effects on the social and psychological aspects of people. The lack of any definitive treatment or preventive method for COVID-19 has caused a great deal of stress and anxiety in people.

Objective The aim of this study was to investigate the demographic characteristics and common complaints of callers to the telephone counseling helpline to receive services for anxiety and stress caused by COVID-19.

Methods This is a descriptive cross-sectional study. The study samples were 1978 callers to the telephone counseling helpline of the Academic Center for Education, Culture and Research (ACECR) in Iran. Data collection was done by a checklist made by researchers and provided to the consultants.

Findings Most of callers (65.8\%) were women and married (77.7\%) with a mean age of 44.14 years; $41.5 \%$ of callers with no any symptoms were afraid and worried about getting infected; $26.6 \%$ stated their anxiety was due to worry that their first-degree relatives may get infected, and $8.5 \%$ reported that their anxiety was because of fear of economic problems and loss of job or income.

Conclusion The main users of the telephone counseling helpline was married women aged 30-39 years. Considering the anxiety and stress caused by COVID-19 outbreak, it seems necessary to provide counseling services.

\section{Extended Abstract}

\section{Introduction}

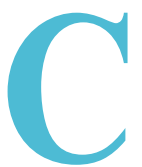

urrently, the COVID-19 which causes acute respiratory syndrome and may lead to the death in some patients, has become pandemic and is spreading to all countries [1]. The first case of coronavirus in Iran was reported on February 18, 2020 in Qom and quickly spread throughout the country. The current mortality rate in the world due to this disease is 7\%, and in Iran it is 6.3\% [2]. Given that the COVID-19 pandemic has affected almost all economic, political, social and even military aspects of the countries in the world, the psychological effects of this viral disease on mental health of people is very important [3]. Previous studies have shown that acute infectious diseases such as SARS can lead to anxiety, depression, and post-traumatic stress disorder [4]. In addition to efforts at various levels to control the disease and for medical treat-

\section{* Corresponding Author:}

Bahareh Kashani Movahhed

Address: Academic Center for Education, Culture and Research, Tehran, Iran.

Tel: +98 (21) 22439873

E-Mail: b.kashanimovahhed@gmail.com 
ments, psychological interventions are inevitable during COVID-19 outbreak. One of the actions taken in this regard is the provision of telephone counseling services. The aim of this study was to investigate the demographic characteristics and the most common complaints among the people seeking telephone consulting services for anxiety and stress caused by COVID-19.

\section{Materials and Methods}

This is descriptive study with cross-sectional design. The study participants were all people who called the telephone counseling helpline of the Academic Center for Education, Culture and Research (ACECR) at Shahid Beheshti University of Medical Sciences for receiving services for Anxiety and Stress caused by COVID-19 outbreak from March 19 to April 19, 2020. The information was collected based on a checklist made by the researchers and provided to the consultants. The checklist surveys demographic information, the main reason for calling the Helpline and the main complaint, and compliance with self-quarantine. Data were analyzed in SPSS V. 21 software.

\section{Results}

In total, from March 19 to April 19, 2020, there was 1978 calls with a duration $>1$ min. The highest number of calls was recorded on March 24 and 28. Consultation time in more than $60 \%$ of the calls was $>15$ minutes with an average duration of $9.52 \mathrm{~min} .65 .8 \%$ of the callers were female and $34.2 \%$ were male. The mean age of callers was 44.14 years; $77.7 \%$ were married; $93.5 \%$ of married callers had children and $6.5 \%$ had no children; and $58.14 \%$ of callers were housewives. Among people who had contacted the helpline with feelings of anxiety and stress caused by the outbreak of COVID-19, the most important complaint was the fear of having COVID-19; 41.5\% had fear-induced anxiety without clinical symptoms. Regarding the reasons for this anxiety, $26.6 \%$ expressed their anxiety due to worry that their first-degree relatives who were currently healthy, may get infected; $8.5 \%$ reported economic problems, $7.5 \%$ reported it because of quarantine and staying home; 7.4\% because their close relatives had tested positive for COVID-19; 5.3\% due to having COVID-19 and fear of death; $2.1 \%$ reported the lack of access to health facilities, and $1.06 \%$ mentioned other reasons.

\section{Discussion}

The purpose of this study was to investigate the demographic characteristics and the most common problems of people called the counseling helpline of ACECR which was launched to provide psychological counseling services in the field of anxiety and stress caused by COVID-19. The highest number of calls was recorded on March 24 and 28, 2020. Studies have shown that these dates coincide with the peak of the disease in Iran and the beginning of the social distancing plan by the national headquarters for fighting corona [5]. According to the results of the present study, it can be stated that the main users of this system was married women aged 30-39 years. The study by Wang et al. also showed that women were more prone to depressive disorders and anxiety at the time of COVID-19 outbreak [6].

The results of this study revealed that most of the callers who were healthy at the time of calling and did not have symptoms of the disease, mentioned the anxiety caused by the possibility of getting infected in themselves and their relatives as the reason for contacting the Helpline. Since the inherent function of anxiety is to protect people from lifethreatening factors, health concerns may arise in the form of anxiety if life-threatening diseases occur. People with high health anxiety tend to misinterpret bodily changes and emotions. Some people seek reassurance from doctors and visit the hospital emergency departments with few symptoms which cause overcrowding in hospitals and create additional burden for medical staff [7]. Therefore, identifying people with high health anxiety and providing counseling services to these people can be effective in controlling the disease.

\section{Ethical Considerations}

\section{Compliance with ethical guidelines}

This study was approved by the Ethics Committee of Shahid Beheshti University of Medical Sciences (Code: 4059-999).

\section{Funding}

This Article was extracted from a research project supported financially by ACECR of Shahid Beheshti University of Medical Sciences.

\section{Authors' contributions}

Methodology, Data analysis, Project administration: Faraj Hoseinian Sarajloo, Bahareh Kashani Movahhed; Investigation: Rosa Rashedi, Hale Fateh, Hamidreza Shahbazpour.

\section{Conflicts of interest}

The authors declared no conflict of interest. 
This Page Intentionally Left Blank 


\title{
ويثَّى هاى جمعيتشناسى و شايع ترين شكايت در تماس تيرندَّان با سامانه مشاوره اضطراب و استرس تلفنى جهاد دانشكَاهى
}

فرج حسينيان سراجهلو' هـ ، رزا راشدى'، هاله فاتح'، حميدرضا شهبازيور'، "بهاره كاشانى موحد' هـ

1. جهاد دانشكاهى، دانشكاه علوميزشكى شهيد بهشتى، تهران، ايران.

\begin{abstract}
רيكيد

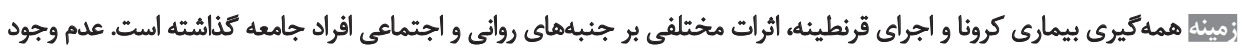

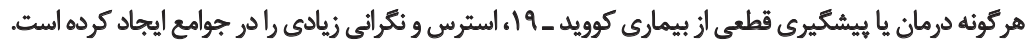

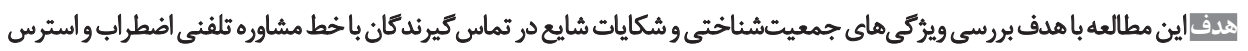
ناشى از بيمارى كرونا انجام شده است.

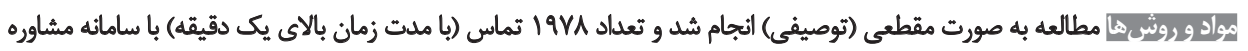

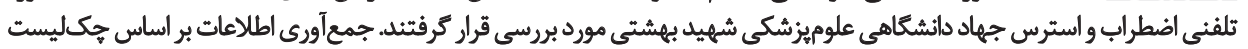

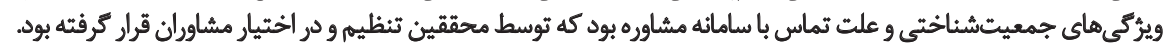

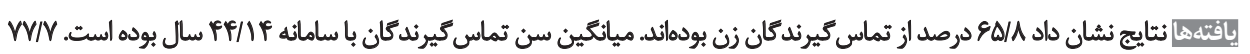

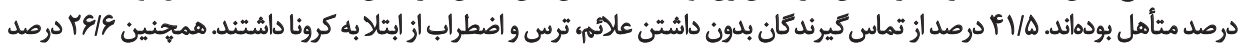

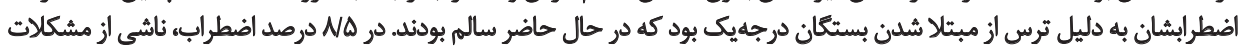
اقتصادى و از دست دادن شغل يا درآمد بودي.

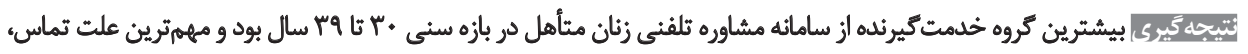

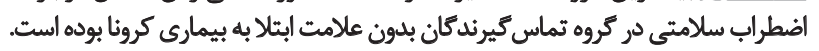

تاريغ دريافت: 9. ارديبهشت 99

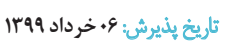

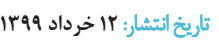

كليدوأوها:

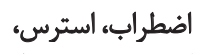

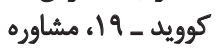
تلفنى

$$
\text { شرقى، آسياى جنوب شرقى، ارويا و اقيانوس آرام غربى [F]. }
$$

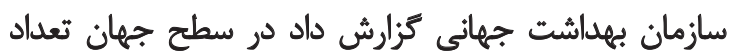

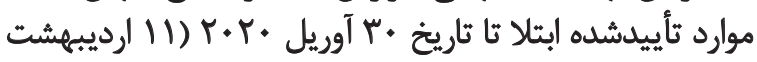

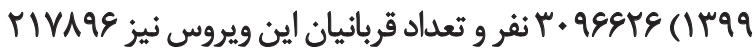

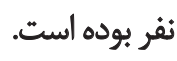

تا زمان نكارش اين مقاله در بين كشورهاى جهان از نظر تعداد

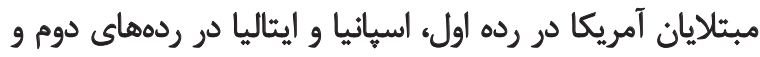

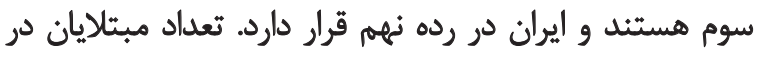

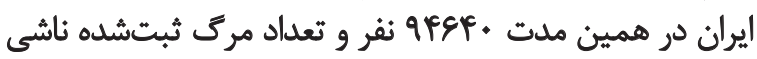

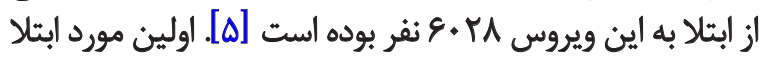

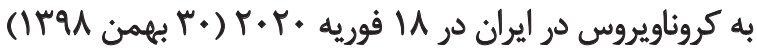

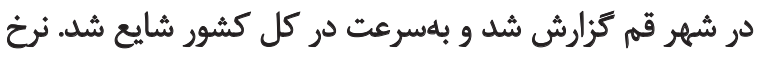

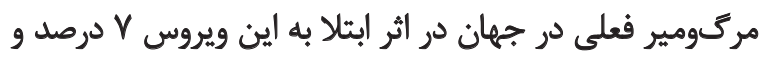

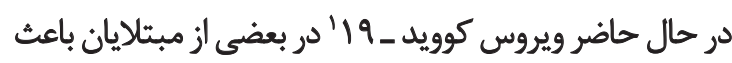

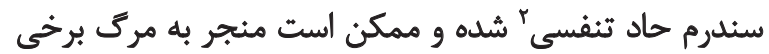

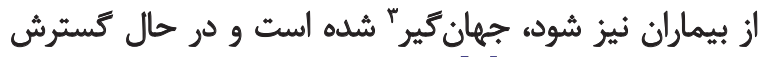

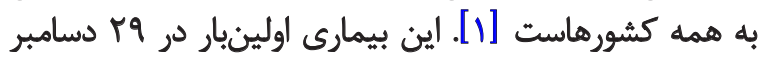

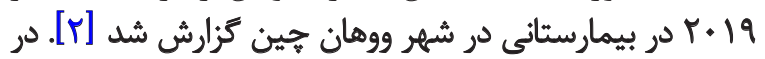

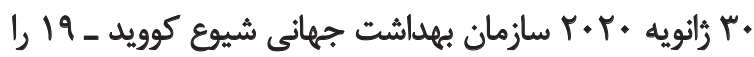

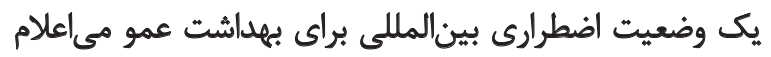

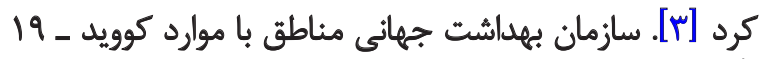
تأييدشده را بدين شرح العلام كرد: آفريقا، قاره آمريكا، مديتران باندانه

1. COVID- 19

2. Acute Respiratory Distress syndrome (ARDS)

3. Pandemic 
اجتنابنايذير است. يكى از اقدامات صورتكرفته در اين زمينه

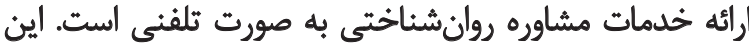

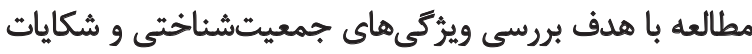

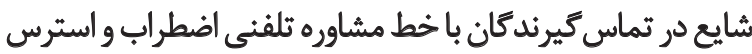

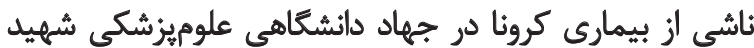
بهشتى انجام شده است.

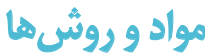

اين مطالعه به صورت مقطعى ـ توصيفى انجام شده است. نمونه

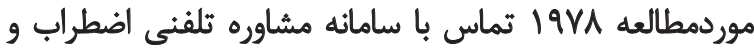

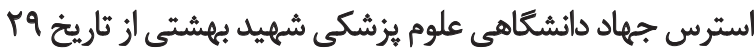

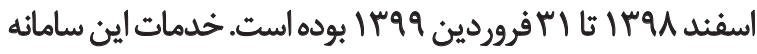

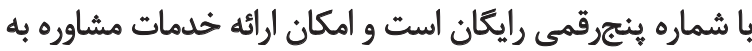

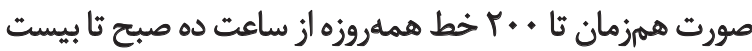

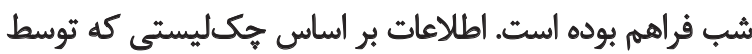

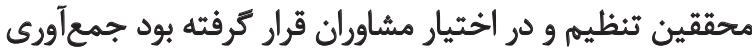

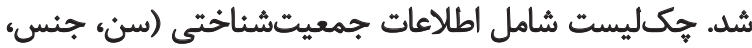

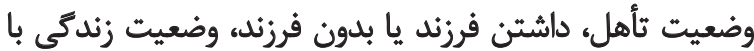

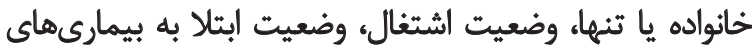

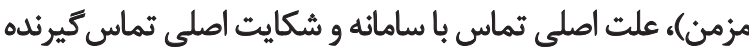

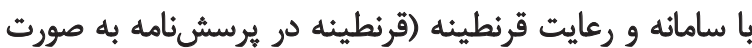

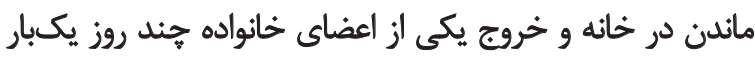
براي خريد مايحتاج ضرورى تعريف شده است) بوده است آنان كليه مشاوران قبل از شروع فعاليت در جلسه آموزشىاي كه به

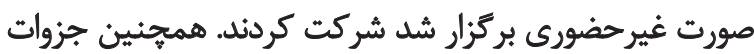

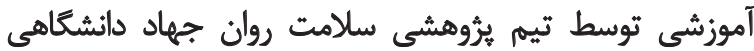
تدوين و در اختيار مشاوران قرار ترفت. تئت

در ابتداي مكالمه به ثماس كيرندكان اعلام مى شيد مكالمه

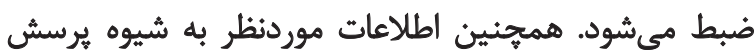

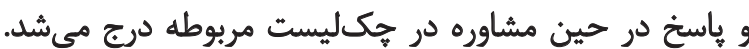

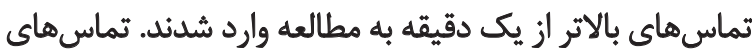

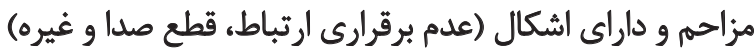

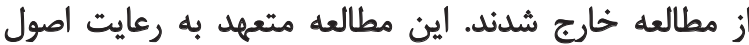

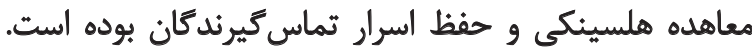

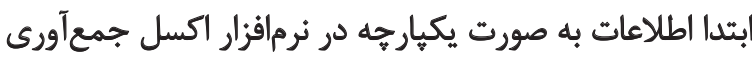
شد. بررسى دادهها به صورت توصيفى و با استفاده از نرمافزار SPSS

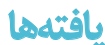

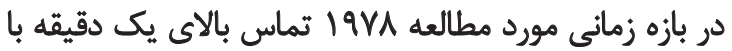

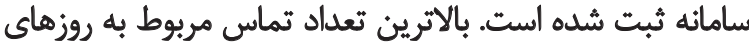

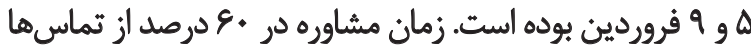

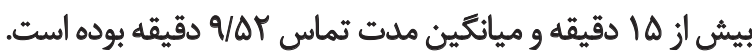

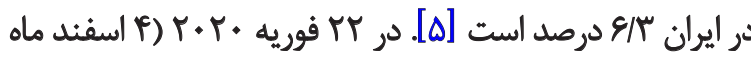

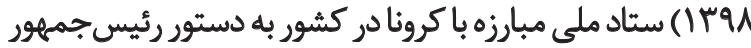

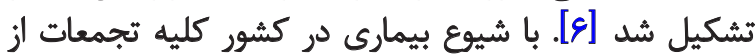

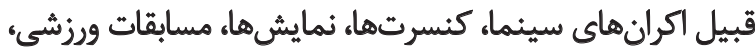

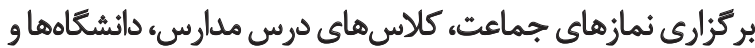

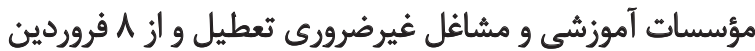

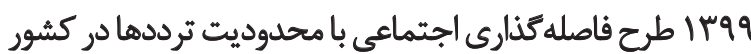

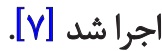

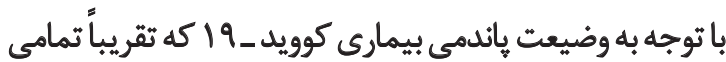

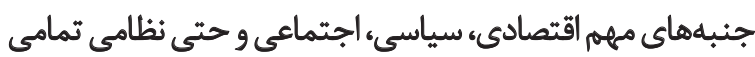

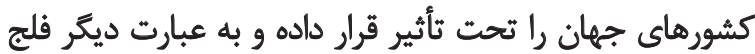

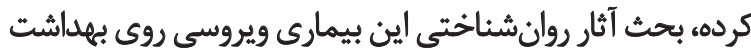

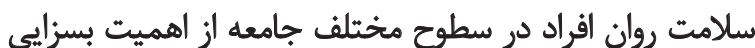

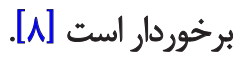

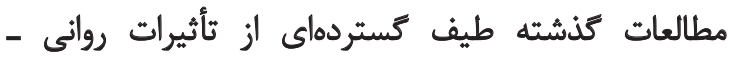
اجتماعى بر افراد رادر سطح فردى، اجتماعى و بيناتئ دالمللى درد

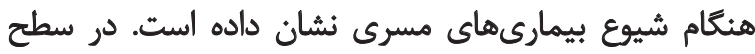

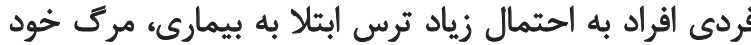

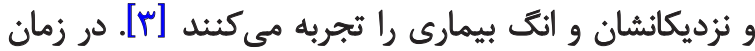

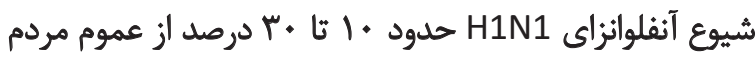

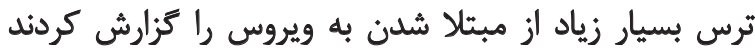

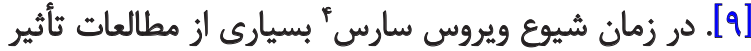

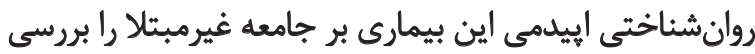

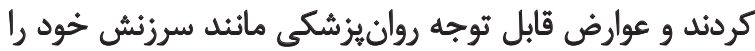

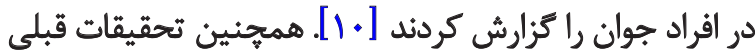

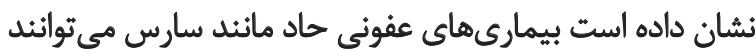

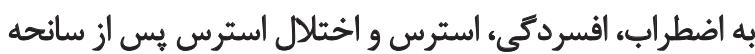

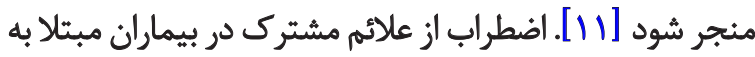

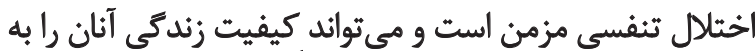

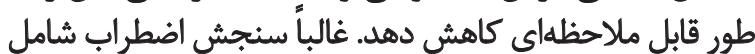

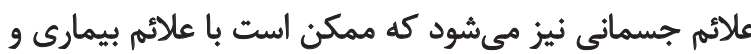

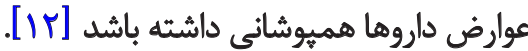

در زمان شيوع بيمارىهاى واكيردار شرايط بُرخطر، غيرقابل

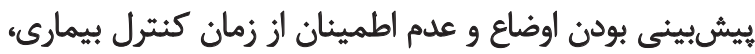

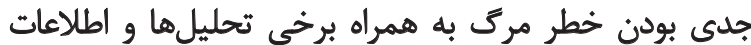

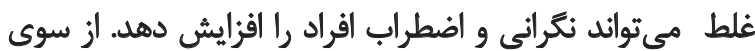

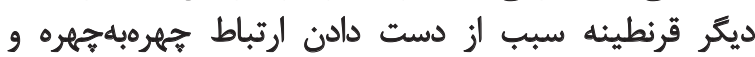

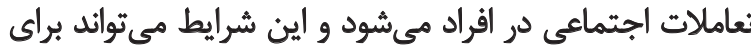

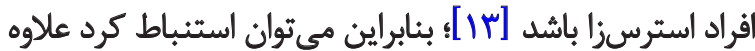

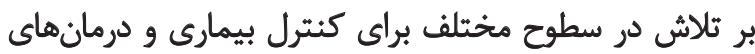
يزشكى، مداخلات روانشناختى در زمان بنان شيوع بيمارى ديمارى

\section{Sars}


جدول ا. اطلاعات جمعيتشئاختي افراد شركت كنينده

\begin{tabular}{|c|c|c|}
\hline تعداد (درصد) & & \\
\hline $\begin{array}{l}\text { SVF }(M T / Y) \\
M+Y(F \Delta / A)\end{array}$ & مرد & جنسيت \\
\hline 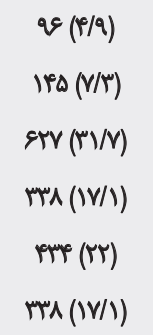 & 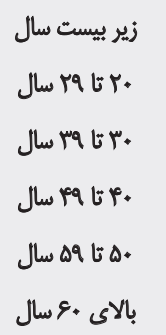 & سن \\
\hline $\begin{array}{l}r \cdot V(I \Delta / \Delta) \\
\text { LOFE }(W / V) \\
W(r / q) \\
\Delta \wedge(r / q)\end{array}$ & 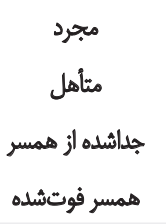 & وضعيت ثأهل \\
\hline $\begin{array}{c}1.9(8 / \Delta) \\
\operatorname{LACT}(9 \% / \Delta)\end{array}$ & بدارون فرزئد & فرزند \\
\hline $\begin{array}{l}\text { WTT (qW/1) } \\
\text { ITE (q/q) }\end{array}$ & با خانواده & نحوهز زندكى \\
\hline $\begin{array}{l}\Delta T A(N V / T) \\
\text { KAF (NF/F) } \\
\text { WLE (ANF) }\end{array}$ & شياغ & وضعيت الشتشال \\
\hline
\end{tabular}

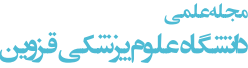

مشاوره يزشكى با سامانه تماس كرفته بودند كه به سامانه •r. F.

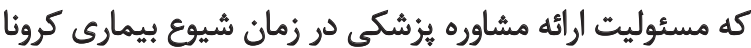

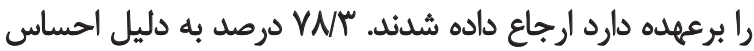

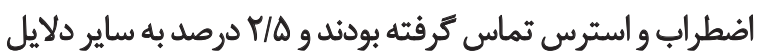

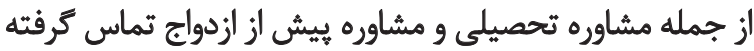

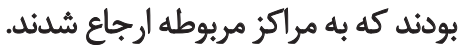

در ميان افرادى كه با احساس اضطراب و استرس ناشى از

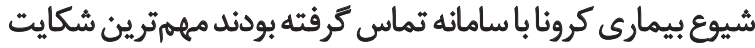

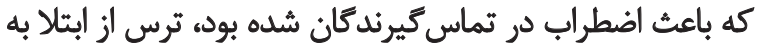

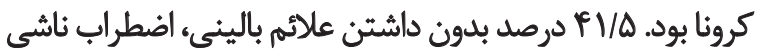

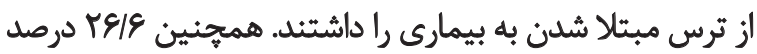

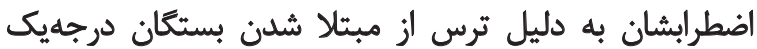

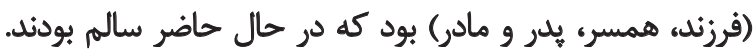

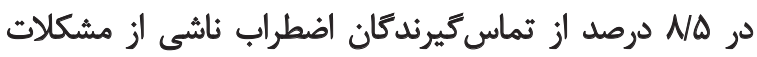
اقتصادى و از دست دادن شغل يا درآمد را كزارش كردئل

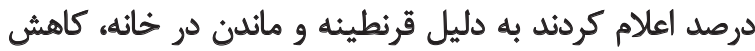

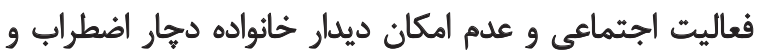

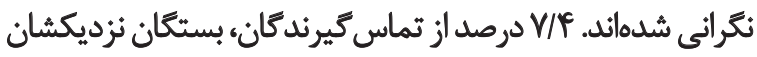

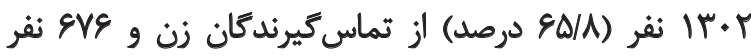

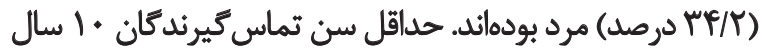

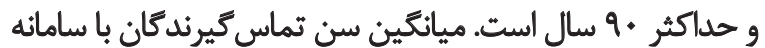

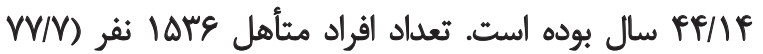
درصد) بود. فرزند (هY بين تماس كيرندكان

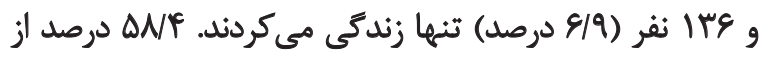
تماس كيرندكان خانهدار بودهاند.

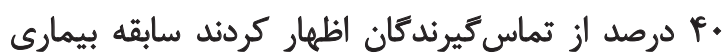

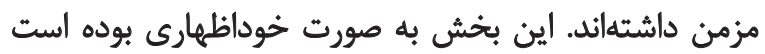

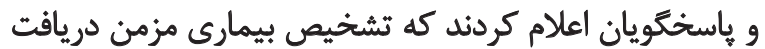

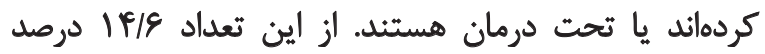

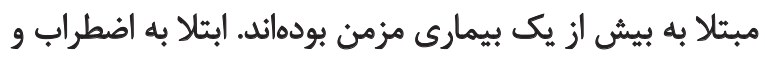

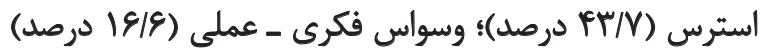

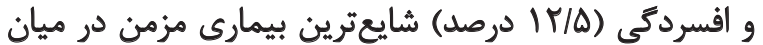
تماس كيرندكان بوده است. 19/T درصد از تماسكيرندكان جهت دريافت اطلاعات و 
از آنجا كه عملكرد ذاتى اضطراب محافظت از افراد در برابر

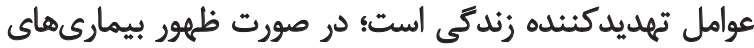

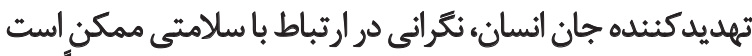

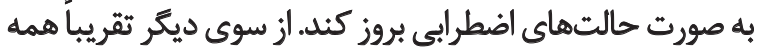

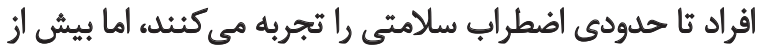

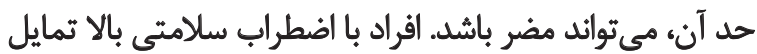

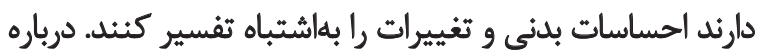

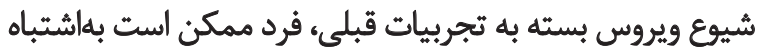

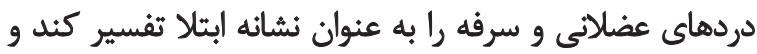

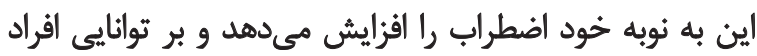

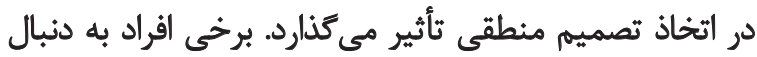

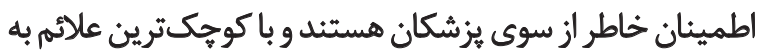

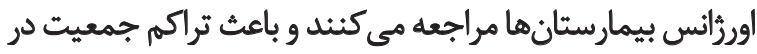

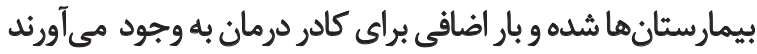

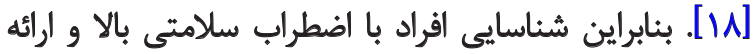
خدمات مشاوره به اين افراد مى تواند در كنترل بيمارى مؤثر باشيد.

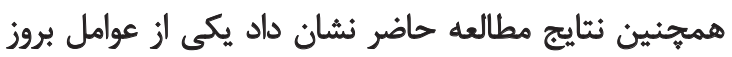
اضطراب كه از سوى تماس كيرند

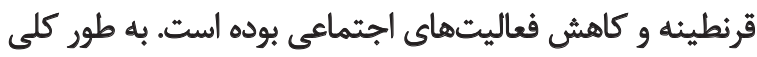

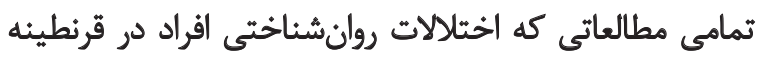

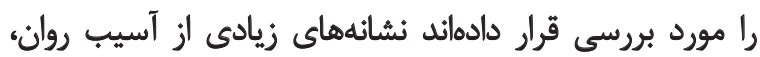

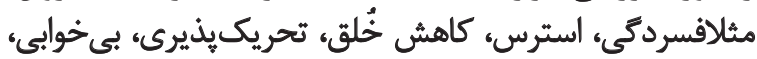

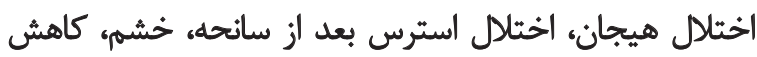

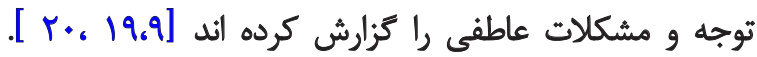

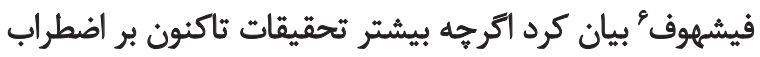

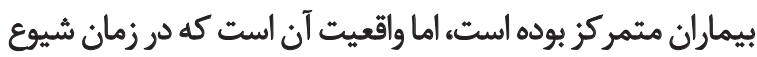

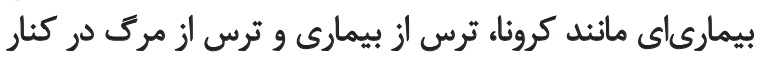

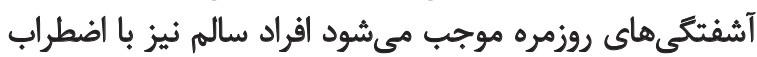

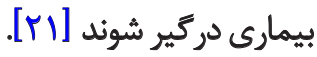

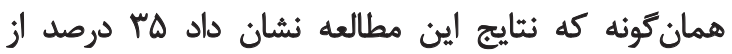

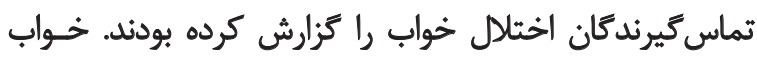

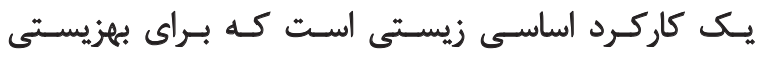

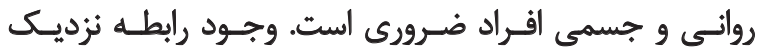

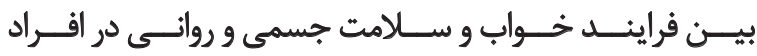

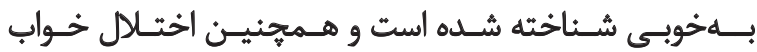

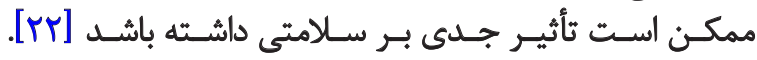

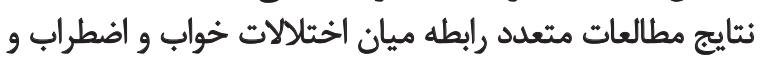

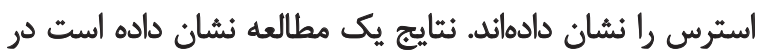

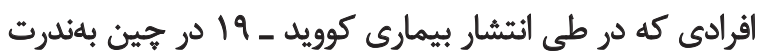

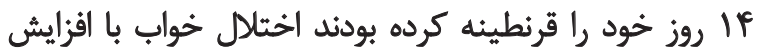

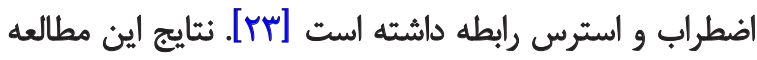

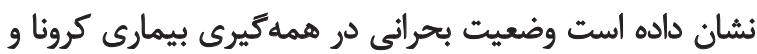

تشخيص كرونا مثبت دريافت كرده بودند و ترس و اضطراب ناشى

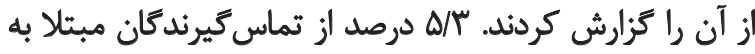

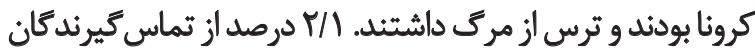

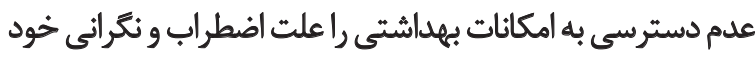

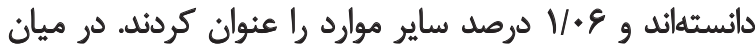

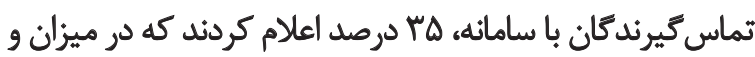

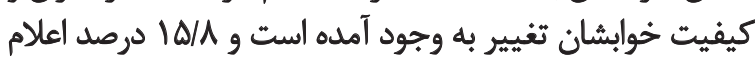

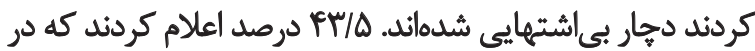
زمان تماس با سامانه در قرنطينه بودداند.

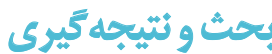

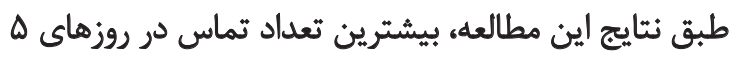

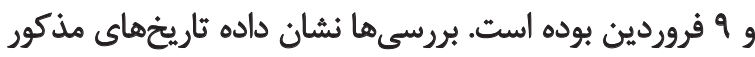

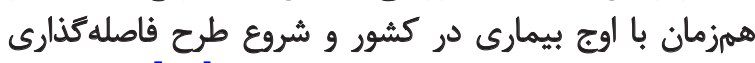

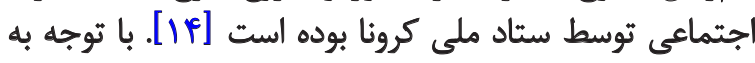

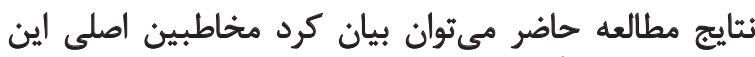

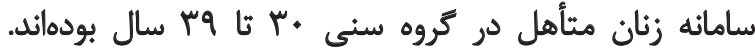

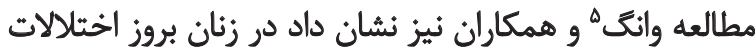

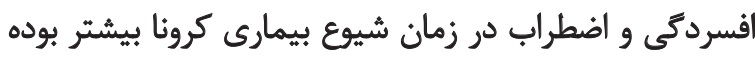

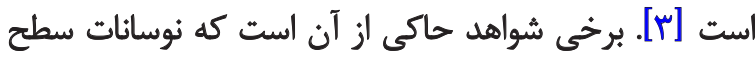

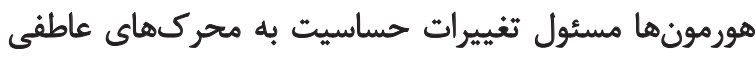

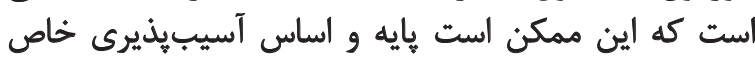

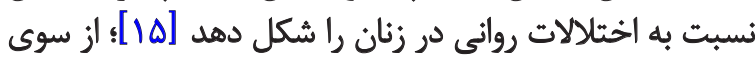

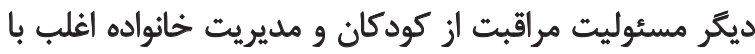

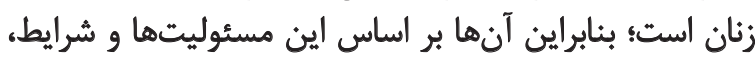

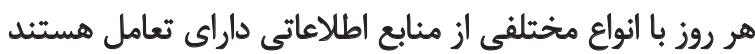

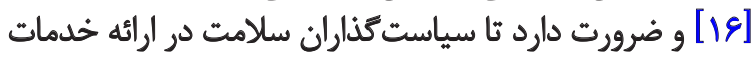

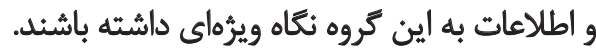

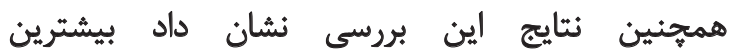

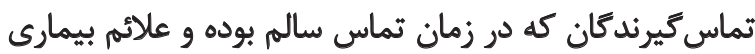

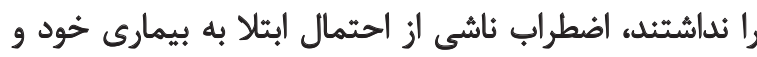

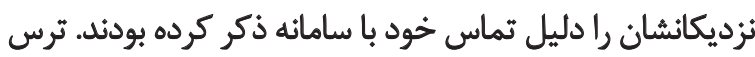

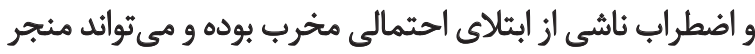

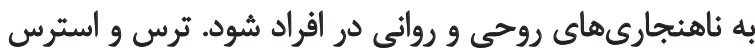

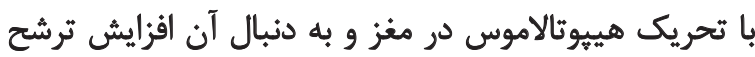

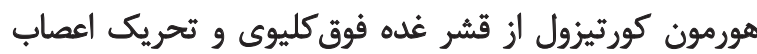

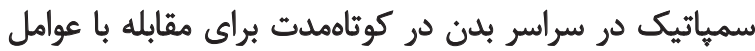

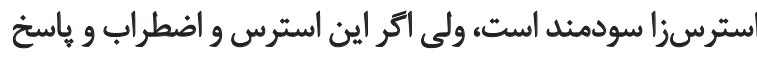

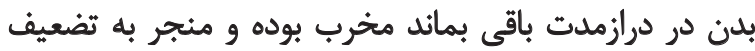

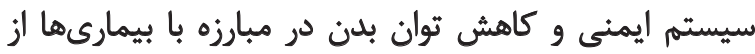

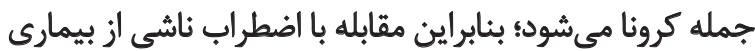

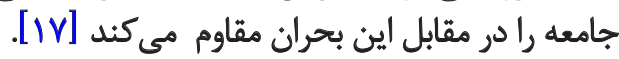


شرايط ويرُهاى كه به تبع آن به وجود مى آيد مىتواند باعث بروز آثار منفى روانى شود.

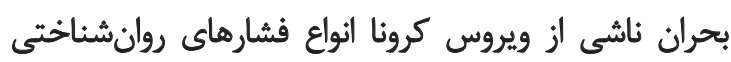

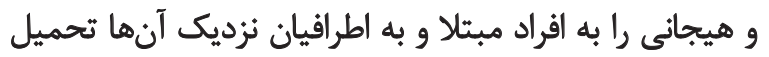

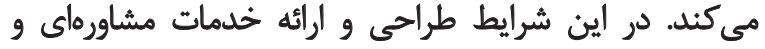

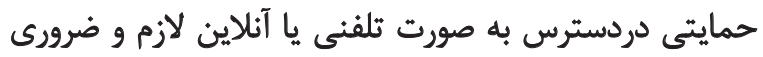

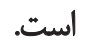

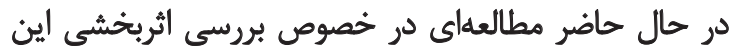

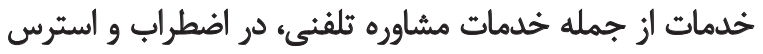

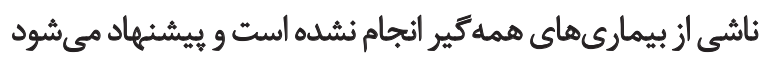

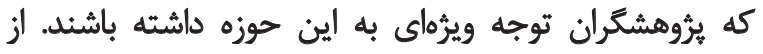

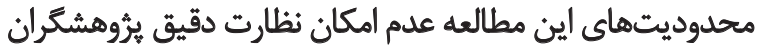

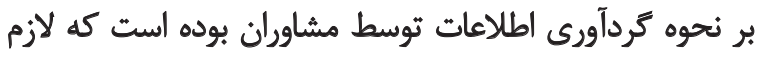

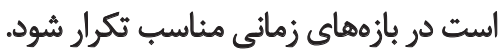

مالاحظاث أغلاق

يبروى از اصول اخلاق يثوهش

اين مطالعه داراى تأييديه اخلاقى از جهاد دانشكاهى

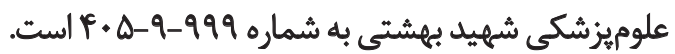

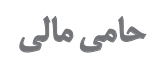

كليه مخارج اين مطالعه بر عهده جهاد دانشكاهي علوميزشكى

$$
\text { شهيد بهشتى بوده است. }
$$

$$
\text { هشاركت نويسندكّان }
$$

مديريت يروره، روششناسى و تحليل داده: فرج حسينيان

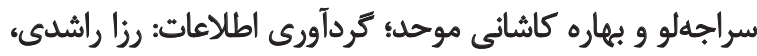
هاله فاتح و حميدرضا شهبازيور.

$$
\text { تعارض منافع }
$$

هيج كونه تضاد منافعى از سوى نويسندكان كزارش نشد. 


\section{References}

[1] Cascella M, Rajnik M, Cuomo A, Dulebohn SC, Di Napoli R. Features, evaluation, and treatment of Coronavirus (COVID-19). In: StatPearls Publishing. StatPearls. Treasure Island, FL: StatPearls Publishing; 2020. [PMID]

[2] Kahn N. New virus discovered by Chinese scientists investigating pneumonia outbreak [Internet]. 2020 [Updated 2020 January 8]. Available from: https://www.wsj.com/articles/ new-virus-discovered-by-chinese-scientists-investigatingpneumonia-outbreak-11578485668

[3] Wang C, Pan R, Wan X, Tan Y, Xu L, Ho CS, et al. Immediate psychological responses and associated factors during the initial stage of the 2019 Coronavirus disease (COVID-19) epidemic among the general population in China. Int J Environ Res Public Health. 2020; 17(5):1729. [DOI:10.3390/ijerph17051729] [PMID] [PMCID]

[4] Alipour A, Ghadami A, Alipour Z, Abdollahzadeh H. Preliminary validation of the Corona Disease Anxiety Scale (CDAS) in the Iranian sample. J Health Psychol. 2020; 8(32):163-75. [In Persian] http://hpj.journals.pnu.ac.ir/article_6571_en.html

[5] World Health Organization. WHO Coronavirus disease (COVID-19) dashboard [Internet]. 2020 [Updated 2020 August 23]. Available from: https://covid19.who.int/

[6] Iranian Studens' News Agency (ISNA). [President orders the Minister of Health to combat and prevent coronary heart disease [Internet]]. 2020 [Updated 2020 February 23]. Available from: https://www.isna.ir/news/98120403112/

[7] Iranian Studens' News Agency (ISNA). [Details of the social separation plan / Travelers return home immediately (Persian) [Internet]. 2020 [Updated 2020 March 26]. Available from: https://www.isna.ir/news/99010703101/ [In Persian]

[8] Li S, Wang Y, Xue J, Zhao N, Zhu T. The impact of COVID-19 epidemic declaration on psychological consequences: A study on active Weibo users. Int J Environ Res Public Health. 2020; 17(6):2032. [DOI:10.3390/ijerph17062032] [PMID] [PMCID]

[9] Rubin GJ, Potts HWW, Michie S. The impact of communications about swine flu (influenza A H1N1v) on public responses to the outbreak: Results from 36 national telephone surveys in the UK. Health Technol Assess. 2010; 14(34):183-266. [DOI:10.3310/hta14340-03] [PMID]

[10] Sim K, Chan YH, Chong PN, Chua HC, Soon SW. Psychosocial and coping responses within the community health care setting towards a national outbreak of an infectious disease. J Psychosom Res. 2010; 68(2):195-202. [DOI:10.1016/j.jpsyy chores.2009.04.004] [PMID] [PMCID]

[11] Xiao H, Zhang Y, Kong D, Li Sh, Yang N. The effects of social support on sleep quality of medical staff treating patients with Coronavirus disease 2019 (COVID-19) in January and February 2020 in China. Med Sci Monit. 2020; 26:e923549. [PMID] [PMCID]

[12] Dong XY, Wang L, Tao YX, Suo XI, Li YC, Liu F, et al. Psychometric properties of the Anxiety Inventory for Respiratory Disease in patients with COPD in China. Int J Chron Obstruct Pulmon Dis. 2016; 12:49-58. [DOI:10.2147/COPD.S117626] [PMID] [PMCID]
[13] Zandifar A, Badrfam R. Iranian mental health during the COVID-19 epidemic. Asian J Psychiatr. 2020; 51:101990. [DOI:10.1016/j.ajp.2020.101990] [PMID] [PMCID]

[14] Corona Headquarters in Tehran Metropolis. All together, until the defeat of Corona [Internet]. 2020 [Updated 2020 March]. Available from: http://tc.sbmu.ac.ir/ [In Persian]

[15] Liu N, Zhang F, Wei C, Jia Y, Shang Z, Sun L, et al. Prevalence and predictors of PTSS during COVID-19 outbreak in China hardest-hit areas: Gender differences matter. Psychiatry Res. 2020; 287:112921. [DOI:10.1016/j.psychres.2020.112921] [PMID] [PMCID]

[16] Urquhart C, Yeoman A. Information behaviour of women: Theoretical perspectives on gender. J Doc. 2010; 66(1):113-39. [DOI:10.1108/00220411011016399]

[17] Allahtavakoli M. Coping with stress of COVID_19 epidemic. J Jiroft Univ Med Sci. 2020; 7(1):253-4. [In Persian] http://journal.jmu.ac.ir/article-1-371-en.html

[18] Asmundson GJG, Taylor S. How health anxiety influences responses to viral outbreaks like COVID-19: What all decisionmakers, health authorities, and health care professionals need to know. J Anxiety Disord. 2020; 71:102211. [DOI:10.1016/j. janxdis.2020.102211] [PMID] [PMCID]

[19] Brooks SK, Webster RK, Smith LE, Woodland L, Wessely S, Greenberg N, et al. The psychological impact of quarantine and how to reduce it: Rapid review of the evidence. Lancet. 2020; 395(10227):912-20. [DOI:10.1016/S0140-6736(20)30460-8]

[20] Wang Y, Xu B, Zhao G, Cao R, He X, Fu S. Is quarantine related to immediate negative psychological consequences during the 2009 H1N1 epidemic? Gen Hosp Psychiatry. 2011; 33(1):75-7. [DOI:10.1016/j.genhosppsych.2010.11.001] [PMID]

[21] Fischhoff B. Speaking of psychology: Coronavirus anxiety [Internet]. 2020 [Updated 2020 February]. Available from: https://www.apa.org/research/action/speaking-of-psychology/ coronavirus-anxiety

[22] Javadi Z, Ghorbani M. The effectiveness of MindfulnessBased Cognitive Therapy (MBCT) on the improvement of sleep quality in patients with generalized anxiety disorder. Horizon Med Sci. 2019; 25(2):117-26. [In Persian] http://hms.gmu. ac.ir/article-1-2945-en.html

[23] Xiao H, Zhang Y, Kong D, Li S, Yang N. Social capital and sleep quality in individuals who self-isolated for 14 days during the coronavirus disease 2019 (COVID-19) outbreak in January 2020 in China. Med Sci Monit. 2020; 26:e923921. [DOI:10.12659/ MSM.923921] [PMID] [PMCID] 
This Page Intentionally Left Blank 